\title{
Management of adverse renal events related to alemtuzumab treatment in multiple sclerosis: a Belgian consensus
}

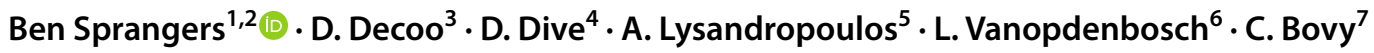

Received: 19 July 2017 / Accepted: 14 November 2017 / Published online: 30 November 2017

(c) The Author(s) 2017. This article is an open access publication

\begin{abstract}
Alemtuzumab is a humanized monoclonal antibody indicated for the treatment of adult patients with relapsing-remitting multiple sclerosis with active disease. Multiple sclerosis (MS) patients treated with alemtuzumab are at increased risk for autoimmune adverse events (thyroid disorders, immune thrombocytopenia, and renal disease). The use of alemtuzumab has been associated with the development of renal immune-mediated adverse events in $0.3 \%$ of patients in clinical trials in MS, which generally occurred within 39 months of the last administration. Both anti-GBM disease and membranous nephropathy have been associated with the use of alemtuzumab. Early detection is necessary to allow for early diagnosis and prevent adverse renal and patient outcomes. Through the implementation of the risk minimization measures, patients can be diagnosed, and treated if needed, early allowing for generally favorable outcomes. This important goal can be reached through health care professional and patient education, careful analysis of the monthly lab tests, and close collaboration between the patient, neurologist, and the nephrologist. This article presents the consensus of Belgian MS specialists and nephrologists on the practicalities of diagnosis, management, and treatment of alemtuzumab-associated renal adverse events based on good clinical practice.
\end{abstract}

Keywords Multiple sclerosis $\cdot$ Alemtuzumab $\cdot$ Renal adverse event $\cdot$ Autoimmunity $\cdot$ Consensus guideline

\section{Introduction}

Alemtuzumab is a humanized monoclonal antibody approved in more than 60 countries for the treatment of multiple sclerosis (MS), and is marketed under the name

Ben Sprangers

ben.sprangers@uzleuven.be

1 Department of Nephrology, University Hospitals Leuven, Herestraat 49, 3000 Leuven, Belgium

2 Laboratory for Experimental Transplantation, Department of Microbiology and Immunology, University of Leuven, Leuven, Belgium

3 Neurology Department, AZ Alma, Gentse Steenweg 132, 8340 Sijsele, Belgium

4 Service de Neurologie, CHU Liège, Esneux, Belgium

5 Neuroimmunology, MS Unit, Neurology Department, CUB, Hôpital Erasme, Route de Lennik 808, 1070 Brussels, Belgium

6 Department of Neurology, AZ Sint Jan Brugge Oostende, Ruddershove 10, 8000 Brugge, Belgium

7 Service de Néphrologie, CHU Sart-Tilman, B35, 4000 Liège, Belgium
Lemtrada ${ }^{\circledR}$. Within the European Union, alemtuzumab is indicated for the treatment of adult patients with relapsing-remitting multiple sclerosis (RRMS) with active disease defined by clinical or imaging features. In clinical trials, alemtuzumab demonstrated superior efficacy compared to high-dose subcutaneous (SC) interferon beta-1a (IFNb-1a) in both treatment-naïve patients and in those with inadequate response to prior therapy, with a consistent and manageable safety and tolerability profile [1]. The most recent efficacy data over 6 years on clinical and MRI lesion activity as well as on brain volume loss suggest that alemtuzumab may provide a unique treatment approach for RRMS patients, offering durable efficacy in the absence of continuous treatment [2].

MS patients treated with alemtuzumab are at increased risk for autoimmune adverse events (AEs) (thyroid disorders, ITP, and renal disease). Two major types of autoimmune renal diseases have been associated with the use of alemtuzumab: membranous nephropathy and anti-glomerular basement membrane disease (anti-GBM disease) [3, 4]. Especially, in anti-GBM disease, early diagnosis is necessary to prevent adverse renal and patient outcomes. The Lemtrada ${ }^{\circledR}$ Risk Management Plan was put in place to ensure early 
detection of symptoms or signs of autoimmune disease, with the aim of minimizing the impacts of alemtuzumab-associated renal effects while maximizing the clinical benefits of the drug with respect to the treatment of RRMS. Renal surveillance includes monthly measurement of serum creatinine and urine analysis with microscopy (detection of red blood cells and proteinuria). In addition, the patient and treating physician are educated to recognize symptoms potentially related to renal disease, such as edema, discolored urine, and hemoptysis $[5,6]$. This monitoring has to be performed for 48 months after the last alemtuzumab administration Nephrology consultation is recommended in the management of nephropathies. This article presents the consensus of Belgian MS specialists and nephrologists on the practicalities of diagnosis, management, and treatment of Lemtrada-associated renal AEs based on good clinical practice.

\section{Alemtuzumab and autoimmune renal disease}

Autoimmune AEs were detected in MS patients treated with alemtuzumab in clinical trials [7]. The 6-year follow-up data of the CARE-MS studies were presented at ECTRIMS 2016 and showed the following frequencies: $39 \%$ of alemtuzumabtreated patients experienced an autoimmune thyroid disorder, $2.6 \%$ an immune thrombocytopenic purpura, and $0.2 \%$ (two cases) an autoimmune renal disease [2]. In post-marketing use through February 2017, 13,000 patients have been treated worldwide with alemtuzumab for MS and the frequency for anti-GBM disease and membranous nephropathy was estimated at $0.13 \%$ [8]. Post-marketing frequencies are not directly comparable to clinical trial incidences because of differences in ascertainment methodology and follow-up duration, and limitations of post-marketing reporting.

\section{Anti-glomerular basement membrane (anti-GBM) disease}

Anti-GBM disease is a rare renal disease caused by the presence of antibodies directed against an antigen located in the glomerular basement membrane (noncollagenous domain 1 of the $\alpha 3$ chain of type IV collagen ( $\alpha 3[\mathrm{IV}] \mathrm{NC} 1)$, resulting in rapidly progressive glomerulonephritis with crescent formation with or without concomitant pulmonary symptoms (hemoptysis, shortness of breath, and cough) $[9,10]$. Crescents are defined as two or more layers of proliferating cells in Bowman's space and are a hallmark of inflammatory glomerulonephritis and a histologic marker of severe glomerular injury. Goodpasture's disease is defined as the presence of glomerulonephritis and pulmonary hemorrhage in the presence of anti-GBM antibodies.
Anti-GBM disease affects approximately $0.5-1.0$ patients per million in the general population. This disease represents around $20 \%$ of cases of rapidly progressive glomerulonephritis and is found in less than 3\% of all kidney biopsies for any reason $[11,12]$. Anti-GBM disease occurs in older children and adults without age preference. Younger patients more often have concurrent pulmonary involvement, than older patients [13,14]. Although anti-GBM disease is most often idiopathic, several triggering factors have been proposed, e.g., infection, smoking, hydrocarbons, smoked cocaine, urinary tract obstruction, and lithotripsy [9, 15-18]. A genetic predisposition to anti-GBM disease has been reported: increased risk associated with HLA-DR15 and DR4 and decreased risk associated with HLA-DR1 and DR7 [19-22]. HLA-DR15 is also associated with an increased risk of MS.

Clinically, patients affected by anti-GBM disease can present with diverse symptoms [general symptoms, edema, hypertension, pulmonary symptoms (dyspnea, hemoptysis)]. General symptoms such as fever, malaise, anorexia, weight loss, and arthralgia may occur but are generally only mild. The outcome of anti-GBM disease is determined by the renal function, the need for dialysis at presentation, and the number of glomeruli affected by crescent formation determined post biopsy. Without immunosuppressive treatment, death or dialysis ensues in more than $90 \%$ of patients with anti-GBM disease [11]. A recent report confirmed oligoanuria as the strongest negative predictor of renal and patient survival. Furthermore, the percentage of glomerular crescents was the only pathologic parameter associated with poor renal outcome in anti-GBM disease [23].

The treatment of anti-GBM disease consists of a combination of plasmapheresis, corticosteroids, and cyclophosphamide. Plasmapheresis will result in a rapid decline of antiGBM antibodies in the circulation, while prednisone and cyclophosphamide are administered to halt antibody production. Almost half of anti-GBM patients will benefit from this treatment by not progressing to dialysis-dependency and survive [24, 25]. Renal recovery is most likely in patients without oligoanuria at the time of diagnosis. Renal recovery is unlikely in patients with $100 \%$ of glomeruli affected on biopsy and with early dialysis-dependency. Early initiation of therapy is most important to optimize renal outcome and long-term prognosis [13].

\section{Membranous nephropathy (MN)}

Membranous nephropathy (MN) is the most common cause of nephrotic syndrome in adults. In this disease, immunoglobulins (IgG) and complement factors deposit in the subepithelial area of the glomerular basement membrane (GBM); between the GBM and the visceral epithelial cells (podocytes). Podocytes are highly specialized cells responsible 
for the protein sieving of the glomerular barrier. Podocytes form interdigitating foot processes covering the GBM. Foot processes are connected through a scale-like protein complex called the "slit-diaphragm". The presence of antibodies and complement fractions in contact with the podocytes leads to their dedifferentiation with foot process effacement and fusion. Consequently, abnormal amounts of protein pass through the glomerular filter from the vascular compartment into the urine. MN symptoms include edema, hypertension, and lab abnormalities: elevated urinary protein, low serum albumin and protein, and dyslipidemia with or without abnormal glomerular filtration rate.

MN may be primary or secondary in nature. Secondary membranous nephropathy can be seen in the context of autoimmune diseases such as systemic lupus erythematosus (class V according to the ISN/RPS classification) or, more rarely, be associated with infectious diseases (hepatitis B, $\mathrm{C}$, parasites, etc.), drugs and toxins (gold, penicillamine, NSAID), or with malignancies (mainly solid tumors, and more common in patients older than 65 years). In $70 \%$ of the cases, MN is primary or idiopathic (pMN). In 2009, the group of David Salant identified the most important antigen involved in the pathogenesis of pMN, the M-type phospholipase A2 receptor (PLA2-R) [26]. This receptor is overexpressed on the membrane of podocytes and may elicit an auto-immunization resulting in the formation of mainly IgG4 anti-PLA ${ }_{2} \mathrm{R}$ antibodies and the subsequent development of membranous nephropathy. Anti-PLA ${ }_{2} \mathrm{R}$ antibodies can be detected in 70-80\% of patients with pMN. In 2014, another podocyte antigen was reported to be involved in the pathogenesis of $\mathrm{pMN}$ : Thrombospondin type-1 domain containing 7A (THSD7A). This antibody is found in approximately $10 \%$ of pMN [27]. For the remaining $10-20 \%$ of patients with $\mathrm{pMN}$, the responsible antigen has not yet been identified.

The outcome for patients with $\mathrm{MN}$ is difficult to predict. Three patterns of clinical evolution have been identified in patients with MN. About one-third will experience spontaneous remission, one-third will remain stable with persisting nephrotic syndrome with no progression to renal failure, and one-third will experience progressive kidney function deterioration necessitating renal replacement therapy (dialysis, renal transplant). Factors that predict poor prognosis (high likelihood of progression to end-stage renal disease) are decreased renal function at diagnosis and heavy proteinuria ( $>8 \mathrm{~g}$ /day) [28-34]. In addition, the titer of anti-PLA ${ }_{2} \mathrm{R}$ antibodies has been reported to be a predictor of outcome [35-37].

The diagnosis and treatment of MN are not a nephrological emergency. If MN is considered secondary, the underlying cause should be treated/removed. First line treatment in all MN patients is symptomatic, anti-proteinuric therapy consisting of ACE inhibitors or antagonists of type II angiotensin receptors in combination with salt restriction and, if necessary, diuretics. Immunosuppression is to be considered for patients with persistent nephrotic syndrome and proteinuria above $4 \mathrm{~g} /$ day after 6 months of anti-proteinuric therapy and/or disabling or life-threatening symptoms related to nephrotic syndrome (thromboembolic events), and/or patients in whom serum creatinine has risen by $30 \%$ or more within 6 months [38]. The recommended immunosuppressive treatment consists of the modified Ponticelli scheme consisting of three 2-monthly cycles of 1 month IV and then oral steroids followed by 1 month oral alkylating agents (cyclophosphamide rather than chlorambucil). Continuous use of oral cyclophosphamide may also be an option. As an alternative, calcineurin inhibitors, cyclosporine + corticosteroid, or tacrolimus monotherapy [13] can be used. In recent years, several groups have reported promising results using rituximab in the treatment of MN [39].

\section{Nephropathies in the context of alemtuzumab treatment}

Anti-GBM disease following alemtuzumab therapy in multiple sclerosis (MS) patients was first reported in 2006 [40]. The first two patients diagnosed with anti-GBM disease related to alemtuzumab treatment both developed to endstage renal disease, highlighting the necessity to actively monitor for signs of renal disease and allow for early diagnosis and treatment. Subsequently, there has been intense renal surveillance during almost all clinical trial and non-trial treatments resulting in substantially complete documentation of, to date, seven cases of nephropathy in total [41-46]. While drug-related nephropathies are well described, and several mechanisms are recognized [46], alemtuzumabrelated nephropathies appear to be a renal manifestation of a general increased tendency of autoimmunity following alemtuzumab treatment. De novo autoimmunity may relate to the proposed mechanism of action of alemtuzumab, i.e., lymphocyte depletion and repopulation. The antibody testing to allow distinction between primary and secondary membranous nephropathy (PLA2-R and THSD7A antibody detection) has not been performed in the patients treated with alemtuzumab. At this moment, we cannot definitely classify these cases of membranous nephropathy as either primary or secondary.

\section{Management of autoimmune renal AEs in the context of alemtuzumab treatment}

Early detection and treatment of nephropathy may decrease the risk of poor outcomes. To minimize the risk of severe disease, regulatory authorities in many countries have 
approved the marketing of Lemtrada ${ }^{\circledR}$ with the implementation of a comprehensive risk minimization plan that includes health care professional (HCP) and patient education and monthly blood and urine analysis monitoring for 48 months after the last alemtuzumab infusion. In addition, during and after this period of time, testing should be performed based on clinical findings suggestive of nephropathies [6].

The practical recommendations in this paper propose a sound approach in case a renal AE is suspected in an alemtuzumab-treated RRMS patient.

Before starting treatment with alemtuzumab: baseline evaluation of renal status and risk of AE.

As stated in the summary of product characteristics (SmPC), serum creatinine levels and urinalysis with microscopy should be obtained prior to initiation of treatment [6]. Furthermore, our consensus recommendation is to perform a determination of urinary protein content before the initiation of alemtuzumab. Abnormalities identified at this time should be discussed with a nephrologist to see whether additional diagnostic evaluation is required. Whether alemtuzumab can be administered in patients with active glomerular disease has not been investigated and should be discussed on an individual basis.

Once alemtuzumab has been administered: monitoring of renal status. The SmPC clearly defines which tests ought to be done and at what frequency: serum creatinine levels and urinalysis with microscopy should be obtained at monthly intervals thereafter for 48 months after the last infusion. The observation of clinically significant changes (i.e., an increase of $30 \%$ or more) from baseline in serum creatinine, unexplained hematuria, and/or proteinuria and symptoms such as edema, should prompt further evaluation for nephropathy including immediate referral to a specialist [6]. It is important to note that small increases in serum creatinine (possibly within the normal range) can correspond to important changes in renal function. Therefore, we consider a relative change of $30 \%$ in serum creatinine compared to baseline creatinine being significant.

In the next six sections, different scenarios based on abnormal lab tests and/or symptoms are presented together with our consensus recommendations for the appropriate actions to be taken. Before discussing the different scenarios, we want to stress that any sign of pulmonary involvement of anti-GBM disease (haemoptysis, exertional dyspnoea) warrants immediate contact with a physician and referral to the emergency room and/or evaluation by a nephrologist the same day. Pulmonary involvement has not been seen with alemtuzumab-related anti-GBM disease in clinical trials with alemtuzumab.

\section{Scenario 1: Isolated microscopic hematuria}

This will probably be the most frequent scenario (Fig. 1). It is important to note that anti-GBM disease can have a subacute course, so isolated microscopic hematuria can be the initial sign of alemtuzumab-related anti-GBM disease. The first action with this finding is to check for the presence of menstruation in female subjects or a urinary tract infection (UTI). When hematuria is microscopic, urine analysis should be repeated weekly to determine whether hematuria persists. When hematuria has disappeared and serum creatinine remains normal at that time, monthly follow-up should be resumed and no further action is required. In case hematuria persists even in association with a normal serum creatinine, the patient should be referred to a routine nephrology consultancy as soon as possible and a test for anti-GBM antibodies should be carried out. When hematuria is associated with an increased serum creatinine, a nephrologist should be contacted immediately and an anti-GBM antibody test should be carried out. This situation is described in more detail in scenario 3 .

\section{Scenario 2: Isolated gross hematuria}

If the patient notices a change in their urine color, they must immediately inform their neurologist/GP who should carry out a non-scheduled blood and urine analysis (Fig. 2). Other causes of red discoloration of the urine should be excluded. Gross hematuria is associated with a risk for bladder obstruction because of blood clots and the occurrence of gross hematuria should, therefore, be discussed with a nephrologist to decide whether an urgent ultrasound examination of the urinary tract should be carried out. Otherwise, the same procedure as described in scenario 1 should be followed.

\section{Scenario 3: Hematuria and elevated serum creatinine! $\rightarrow$ emergency}

A significant increase of serum creatinine $(+30 \%$ from baseline) or a decrease in the glomerular filtration rate (GFR) should prompt immediate action (Fig. 3). Immediate discussion with a nephrologist and biochemical retesting is required. The nephrologist will decide the need for immediate consultation and hospitalization, and whether a kidney biopsy is indicated.

\section{Scenario 4: Isolated proteinuria}

When significant proteinuria is detected $(>0.5 \mathrm{~g} /$ day or on dipstick $>1+$ ), we recommend determining proteinuria on a random urine sample and have proteinuria expressed in $\mathrm{g} / \mathrm{g}$ creatinine (Fig. 4). A result for proteinuria below $0.2 \mathrm{~g} / \mathrm{g}$ creatinine is normal and does not need further investigations. In this circumstance, normal monthly monitoring should be resumed. A result for proteinuria above $0.2 \mathrm{~g} / \mathrm{g}$ creatinine needs further investigation. When a UTI 
Fig. 1 Isolated microscopic hematuria

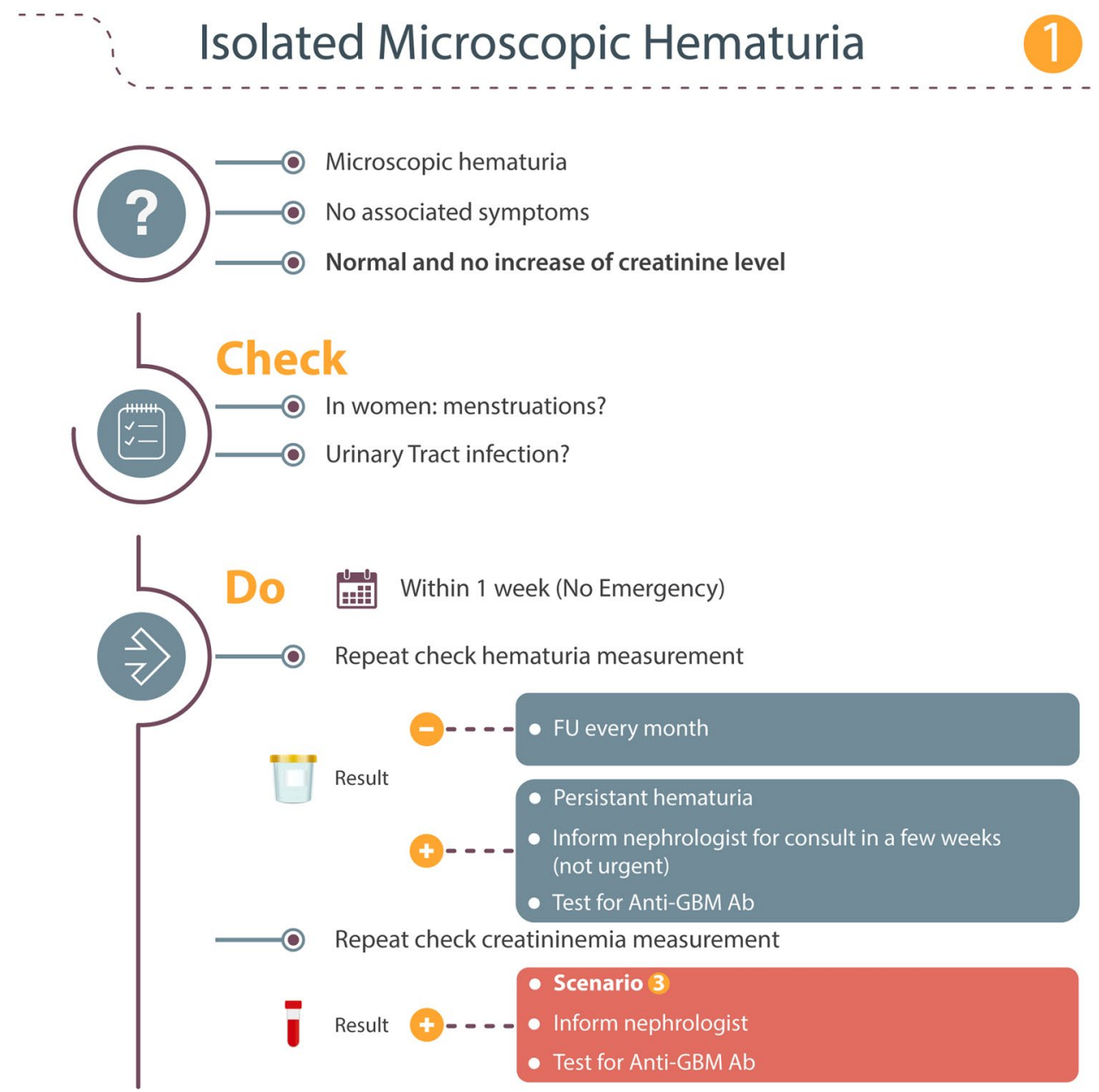

is excluded, patients with pathologic proteinuria should be referred to a nephrologist for a non-urgent consultation.

\section{Scenario 5: Subjects with renal symptoms}

Subjects treated with alemtuzumab are educated to be vigilant for symptoms of possible kidney disorders (Fig. 5). These include red-discoloration of the urine and swelling of the legs, feet, or eyelids. In addition, coughing up blood is an alarm symptom for which patients should immediately contact their neurologist or seek immediate medical attention as it may signal underlying pulmonary damage/ bleeding [6]. When a patient observes such symptoms in between the monthly lab testing, the following approach is proposed:

1. Perform lab testing on urine and blood.

2. Act according to the results as described in the other scenarios.

\section{Scenario 6: Isolated elevated serum creatinine}

The finding of an elevated serum creatinine without hematuria or proteinuria is unlikely to require immediate action (Fig. 6). Anti-GBM disease is always accompanied with hematuria, and the predominant presentation of $\mathrm{MN}$ is proteinuria. Of course, it is important to evaluate the patient for other causes of increased serum creatinine such as reduced fluid intake, (undetected) cardiac disease, or unadjusted diuretics dosage. A non-urgent consultation with a nephrologist is advised.

\section{Discussion}

The use of alemtuzumab has been associated, albeit rarely, with the development of immune-mediated renal adverse events. Membranous nephropathy is not a nephrologic emergency, but requires intensive follow-up and treatment. Anti-GBM disease is potential cause of life-threatening 
Fig. 2 Isolated gross hematuria

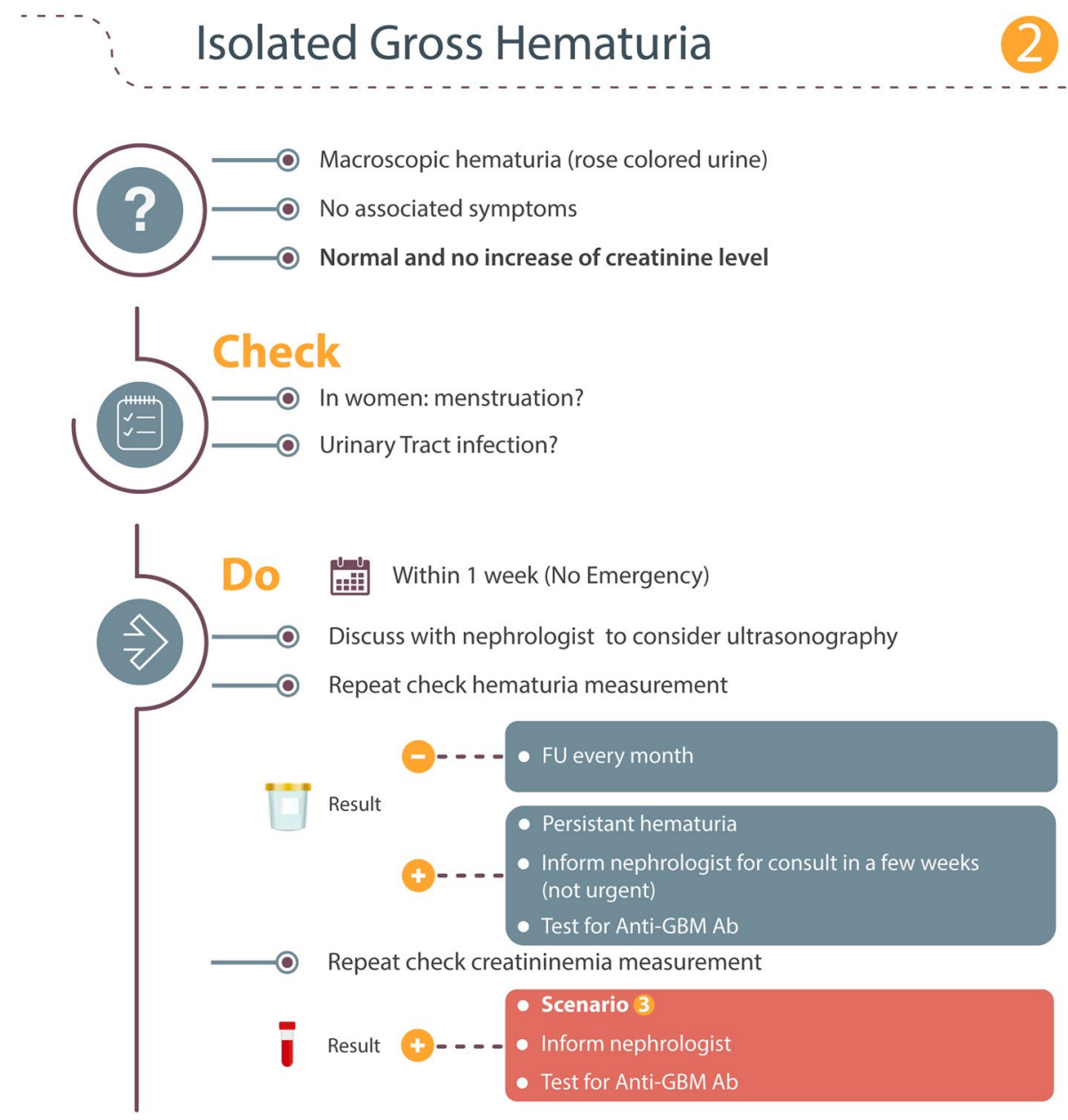

Fig. 3 Microscropic hematuria and elevated serum creatinine

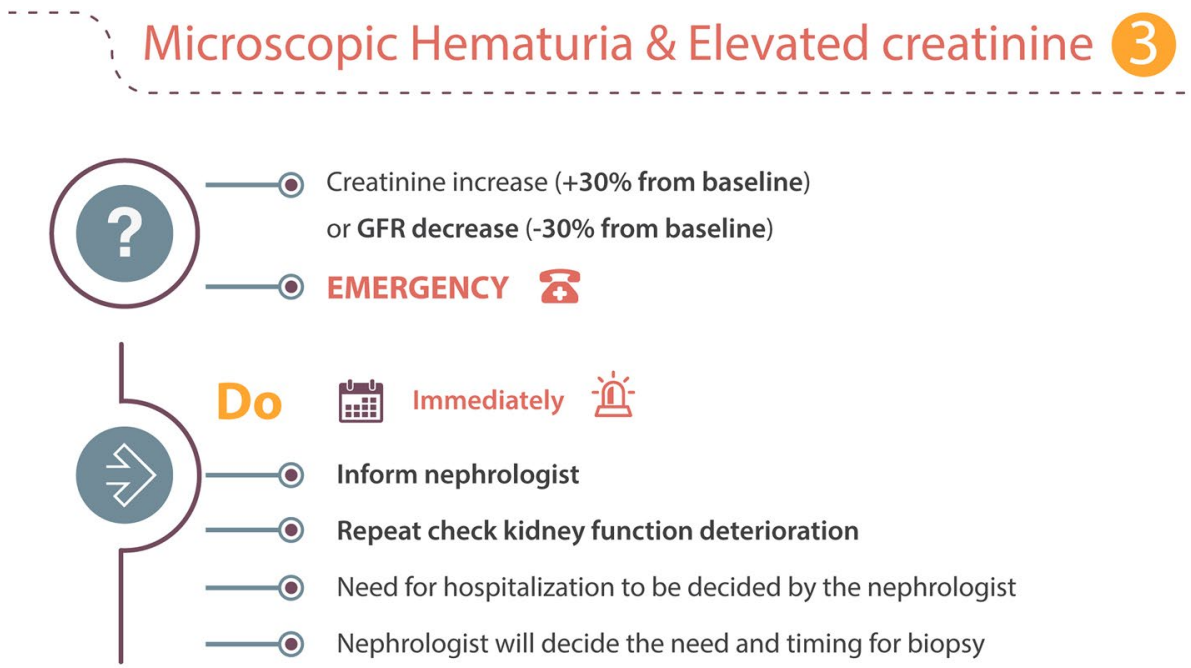

acute renal failure and requires immediate treatment. AntiGBM disease following alemtuzumab therapy in multiple sclerosis (MS) patients was first reported in 2006 [40]. In response, Sanofi Genzyme implemented mitigation strategies in the clinical development program. In the post marketing setting, according to the label, a monthly blood and urine analysis until 48 months after the last alemtuzumab infusion is mandatory to capture potential autoimmune AEs in their preclinical stage. 
Fig. 4 Isolated proteinuria

\section{: Isolated Proteinuria}

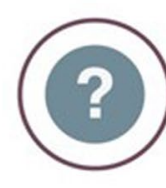

- Microscopic hematuria

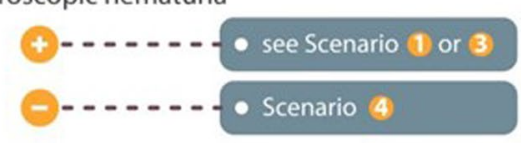

Significant proteinuria: $>0,5 \mathrm{~g} / \mathrm{d}$ or dipstick $>1+$

- Normal and no increase of creatinine level

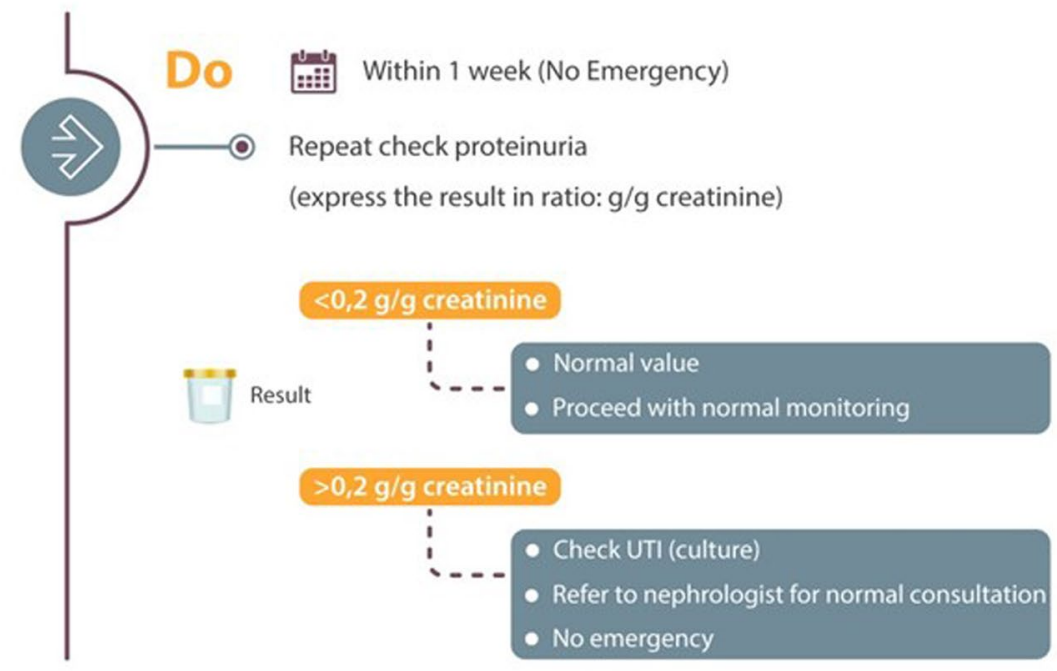

Fig. 5 Renal symptoms

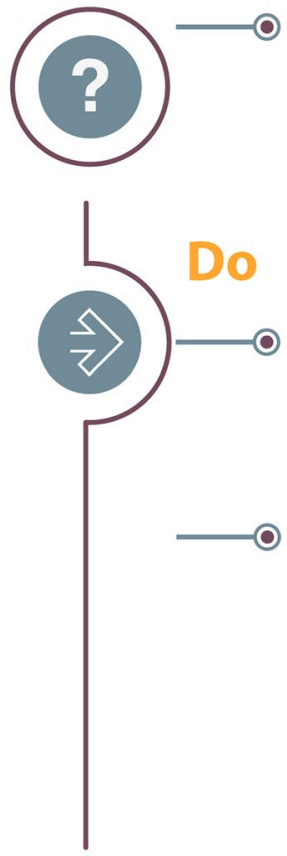

Renal symptoms in between monthly lab testing

i Blood in the urine (urine may be red or tea-coloured)

- Swelling in legs or feet (oedema)
- Blood in the urine (urine may be

- New hypertension or coughing up blood (hemoptysis

Perform Lab testing

Urine microscopy \& analysis

- Creatininemia

Act according to results, see other scenarios

Emergency?

Yes, when creatininemia is increased $\rightarrow$ Scenario 3

- Yes, in case of symptoms of uremia (dyspnoea)

- Yes, in case of hemoptysis

Inform Nephrologist immediately 
Fig. 6 Isolated rise in serum creatinine

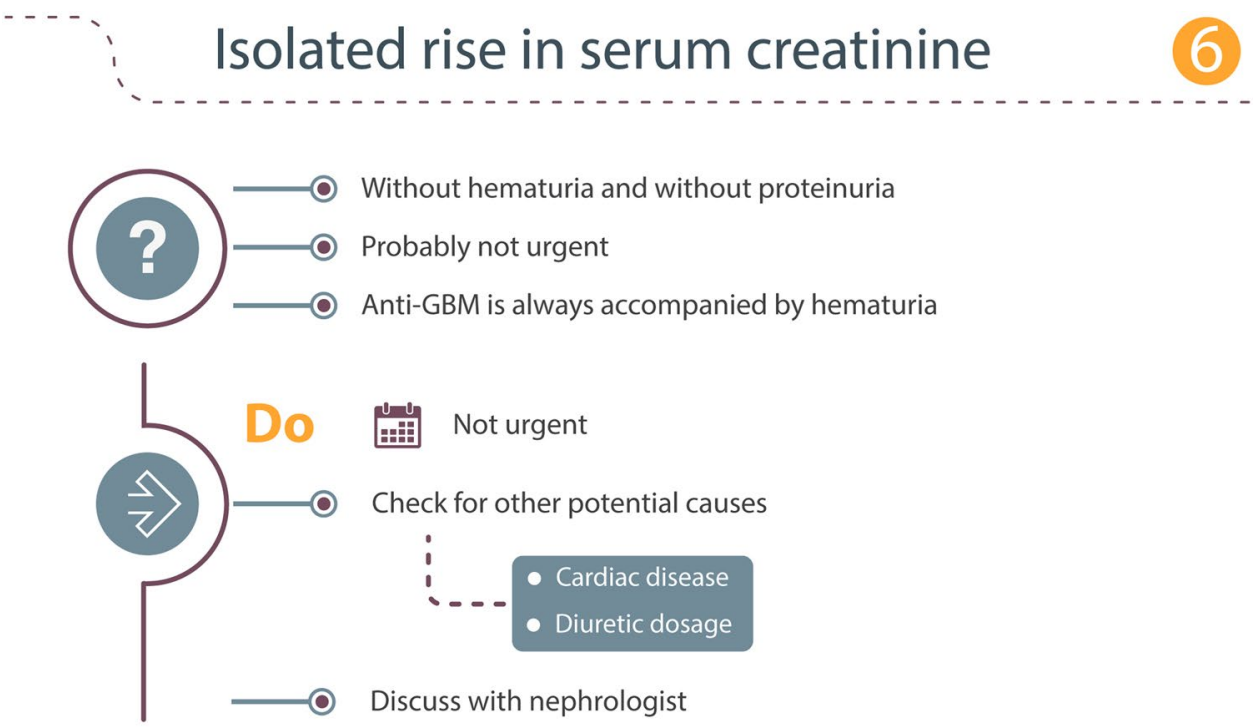

Initial treatment of membranous nephropathy is symptomatic and directed at the reduction of edema and proteinuria. In patients with renal abnormalities and positive anti-GBM antibodies, this treatment should be initiated without delay. The standard treatment of anti-GBM disease consists of plasmapheresis, cyclophosphamide, and steroids.

In this consensus article, we have presented six different scenarios of renal abnormalities that may be encountered and the appropriate actions to take for each scenario.

\section{Conclusion}

The use of alemtuzumab has been associated with the development of renal immune-mediated adverse events in $0.3 \%$ of patients in clinical trials in MS, which generally occurred within 39 months of the last administration. Through the implementation of the risk minimisation measures, patients can be diagnosed, and treated if needed, early allowing for generally favorable outcomes. This important goal can be reached through HCP and patient education, careful analysis of the monthly lab tests, and close collaboration between the patient, neurologist, and the nephrologist. Moreover, collaboration between the neurologist and nephrologist could be facilitated through the pre-establishment of a physician reference network. In this report, we have described different scenarios of renal abnormalities that may be encountered following the administration of alemtuzumab to RRMS patients, and the appropriate actions to take for each.

\section{Compliance with ethical standards}

Conflict of interest The authors declare that they have no conflict of interest.
Disclosures \& disclaimer AL participated as an MS specialist prior to his employment by Sanofi. Editorial support was provided by Thomas Savoye, MD, Blue Jibe Medical Affairs, Herent, Belgium. This article was reviewed by Darren P. Baker, PhD, Marianne Berrens, MD, Chantal Maertens, PharmD, PhD, and Steve Cavalier MD of Sanofi as a courtesy, with no obligation on the authors' part to revise the manuscript. Funding for this editorial support was provided by Sanofi.

Ethical approval This article does not contain any studies with human participants or animals performed by any of the authors.

Informed consent None.

Open Access This article is distributed under the terms of the Creative Commons Attribution 4.0 International License (http://creativecommons.org/licenses/by/4.0/), which permits unrestricted use, distribution, and reproduction in any medium, provided you give appropriate credit to the original author(s) and the source, provide a link to the Creative Commons license, and indicate if changes were made.

\section{References}

1. Berger T, Elovaara I, Fredrikson S et al (2017) Alemtuzumab use in clinical practice: recommendations from European multiple sclerosis experts. CNS Drugs 31:33-50

2. Dayan C, Cuker A, LaGanke C et al (2016) Autoimmunity in patients treated with alemtuzumab for relapsing-remitting multiple sclerosis: 6-year follow-up of the CARE-MS studies. ECTRIMS Online Libr 147015:20

3. Clatworthy MR, Wallin EF, Jayne DR (2008) Anti-glomerular basement membrane disease after alemtuzumab. N Engl J Med 359:768-769

4. Cossburn M, Pace AA, Jones J et al (2011) Autoimmune disease after alemtuzumab treatment for multiple sclerosis in a multicenter cohort. Neurology 77:573-579

5. Willis MD, Robertson NP (2015) Alemtuzumab for the treatment of multiple sclerosis. Ther Clin Risk Manag 11:525-534 
6. European Medicines Agency (2016) Lemtrada (alemtuzumab): product information. 12 Dec 2016

7. Havrdova E, Horakova D, Kovarova I (2015) Alemtuzumab in the treatment of multiple sclerosis: key clinical trial results and considerations for use. Ther Adv Neurol Disord 8:31-45

8. Selmaj KW, Habek M, Bass A et al (2017) Efficacy and safety of alemtuzumab in patients with RRMS is durable over 10 years: follow-up from the CAMMS223 study. Neurology 88:5338

9. Pusey CD (2003) Anti-glomerular basement membrane disease. Kidney Int 64:1535-1550

10. McAdoo SP, Pusey CD (2017) Anti-glomerular basement membrane disease. Clin J Am Soc Nephrol 12(7):1162-1172

11. Wilson CB, Dixon FJ (1973) Anti-glomerular basement membrane antibody-induced glomerulonephritis. Kidney Int 3:74-89

12. Bolton WK (1996) Goodpasture's syndrome. Kidney Int 50:1753-1766

13. Savage CO, Pusey CD, Bowman C et al (1986) Antiglomerular basement membrane antibody mediated disease in the British Isles 1980-4. Br Med J (Clin Res Ed) 292:301-304

14. Levy JB, Hammad T, Coulthart A et al (2004) Clinical features and outcome of patients with both ANCA and anti-GBM antibodies. Kidney Int 66:1535-1540

15. Umekawa T, Kohri K, Yoshioka K et al (1994) Production of anti-glomerular basement membrane antibody after extracorporeal shock wave lithotripsy. Urol Int 52:106-108

16. Bombassei GJ, Kaplan AA (1992) The association between hydrocarbon exposure and anti-glomerular basement membrane antibody-mediated disease (Goodpasture's syndrome). Am J Ind Med 21:141-153

17. Donaghy M, Rees AJ (1983) Cigarette smoking and lung haemorrhage in glomerulonephritis caused by autoantibodies to glomerular basement membrane. Lancet 2:1390-1393

18. Garcia R, Garcia BF, Puras AM (1997) Gil. Pulmonary hemorrhage and antiglomerular basement membrane antibody-mediated glomerulonephritis after exposure to smoked cocaine (crack): a case report and review of the literature. Pathol Int 47:692-697

19. Phelps RG, Rees AJ (1999) The HLA complex in Goodpasture's disease: a model for analyzing susceptibility to autoimmunity. Kidney Int 56:1638-1653

20. Yang R, Cui Z, Zhao J et al (2009) The role of HLA-DRB1 alleles on susceptibility of Chinese patients with anti-GBM disease. Clin Immunol 133:245-250

21. Kitagawa W, Imai H, Komatsuda A et al (2008) The HLADRB $1 * 1501$ allele is prevalent among Japanese patients with anti-glomerular basement membrane antibody-mediated disease. Nephrol Dial Transplant 23:3126-3129

22. Fisher M, Pusey CD, Vaughan RW et al (1997) Susceptibility to anti-glomerular basement membrane disease is strongly associated with HLA-DRB1 genes. Kidney Int 51:222-229

23. Alchi B, Griffiths M, Sivalingam M et al (2015) Predictors of renal and patient outcomes in anti-GBM disease: clinicopathologic analysis of a two-centre cohort. Nephrol Dial Transplant 30:814-821

24. Madore F, Lazarus JM, Brady HR (1996) Therapeutic plasma exchange in renal diseases. J Am Soc Nephrol 7:367-386

25. Couser WG (1988) Rapidly progressive glomerulonephritis: classification, pathogenetic mechanisms, and therapy. Am J Kidney Dis 11:449-464

26. Beck LH Jr, Bonegio RG, Lambeau G et al (2009) M-type phospholipase $\mathrm{A} 2$ receptor as target antigen in idiopathic membranous nephropathy. N Engl J Med 361:11-21
27. Tomas NM, Beck LH Jr, Meyer-Schwesinger C et al (2014) Thrombospondin type-1 domain-containing 7A in idiopathic membranous nephropathy. N Engl J Med 371:2277-2287

28. Honkanen E (1986) Survival in idiopathic membranous glomerulonephritis. Clin Nephrol 25:122-128

29. Glassock RJ (2003) Diagnosis and natural course of membranous nephropathy. Semin Nephrol 23:324-332

30. Cattran DC (2001) Idiopathic membranous glomerulonephritis. Kidney Int 59:1983-1994

31. Cattran D (2005) Management of membranous nephropathy: when and what for treatment. J Am Soc Nephrol 16:1188-1194

32. Pei Y, Cattran D, Greenwood C (1992) Predicting chronic renal insufficiency in idiopathic membranous glomerulonephritis. Kidney Int 42:960-966

33. Cattran DC, Pei Y, Greenwood CM et al (1997) Validation of a predictive model of idiopathic membranous nephropathy: its clinical and research implications. Kidney Int 51:901-907

34. Fervenza FC, Sethi S, Specks U (2008) Idiopathic membranous nephropathy: diagnosis and treatment. Clin J Am Soc Nephrol 3:905-919

35. Kanigicherla D, Gummadova J, McKenzie EA et al (2013) AntiPLA2R antibodies measured by ELISA predict long-term outcome in a prevalent population of patients with idiopathic membranous nephropathy. Kidney Int 83:940-948

36. Hofstra JM, Debiec H, Short CD et al (2012) Antiphospholipase A2 receptor antibody titer and subclass in idiopathic membranous nephropathy. J Am Soc Nephrol 23:1735-1743

37. Hoxha E, Thiele I, Zahner G et al (2014) Phospholipase A2 receptor autoantibodies and clinical outcome in patients with primary membranous nephropathy. J Am Soc Nephrol 25:1357-1366

38. Kidney Disease: Improving Global Outcomes (KDIGO) Glomerulonephritis Work Group (2012) KDIGO clinical practice guideline for glomerulonephritis. Kidney Int Suppl 2:139-274

39. Ruggenenti P, Cravedi P, Chianca A et al (2012) Rituximab in idiopathic membranous nephropathy. J Am Soc Nephrol 23:1416-1425

40. Coles AJ, Cox A, Le PE et al (2006) The window of therapeutic opportunity in multiple sclerosis: evidence from monoclonal antibody therapy. J Neurol 253:98-108

41. Coles AJ, Compston DA, Selmaj KW et al (2008) Alemtuzumab vs. interferon beta-1a in early multiple sclerosis. N Engl J Med 359:1786-1801

42. Cohen JA, Coles AJ, Arnold DL et al (2012) Alemtuzumab versus interferon beta 1a as first-line treatment for patients with relapsing-remitting multiple sclerosis: a randomised controlled phase 3 trial. Lancet 380:1819-1828

43. Coles AJ, Twyman CL, Arnold DL et al (2012) Alemtuzumab for patients with relapsing multiple sclerosis after disease-modifying therapy: a randomised controlled phase 3 trial. Lancet 380:1829-1839

44. Meyer D, Coles A, Oyuela P et al (2013) Case report of antiglomerular basement membrane disease following alemtuzumab treatment of relapsing-remitting multiple sclerosis. Mult Scler Relat Disord 2:60-63

45. Wynn DR, Arnold DL, Coles A et al (2013) Detection, incidence, and management of glomerulonephritis in the alemtuzumab clinical development program. Mult Scler 19:597

46. Perazella MA (2005) Drug-induced nephropathy: an update. Expert Opin Drug Saf 4:689-706 